\title{
Dynamically Stabilized Bright Solitons in a Two-Dimensional Bose-Einstein Condensate
}

\author{
Hiroki Saito and Masahito Ueda \\ Department of Physics, Tokyo Institute of Technology, Tokyo 152-8551, Japan
}

(Dated: October 27, 2018)

\begin{abstract}
We demonstrate that matter-wave bright solitons can be stabilized in 2D free space by causing the strength of interactions to oscillate rapidly between repulsive and attractive by using, e.g., Feshbach resonance.
\end{abstract}

PACS numbers: 03.75.Fi, 05.30.Jp, 05.45.Yv, 34.50.-s

A pendulum with an oscillating pivot can have a stable configuration with its bob situated above the pivot (the inverted pendulum [1]). This well-known but surprising phenomenon is attributed to a net stabilizing force produced by alternating stabilizing and destabilizing forces at a frequency much faster than the natural oscillation frequency of the fixed-pivot pendulum. Similar physics has been employed to focus beams of charged particles in a synchrotron (alternating-gradient focusing 22) and to trap ions in the Paul trap [3]. In this Letter, we present a novel application of such a stabilizing mechanism to producing bright solitons in a two-dimensional (2D) Bose-Einstein condensate (BEC). By a 'bright' soliton we mean a stable, solitary wave whose density is greater than the background one.

In $1 \mathrm{D}$, when the interatomic interaction is attractive, bright solitons can be stabilized even without a trapping potential [4, 5]. In 2D or 3D free space, however, the kinetic pressure and the attractive force cannot balance, and the condensate always collapses or expands. This can be understood by the following simple argument. When the characteristic size of the condensate is $R$, the kinetic and interaction energies are proportional to $R^{-2}$ and $-R^{-d}$ in $d$ dimensions, and an effective potential for $R$ is the sum of these energies. The effective potential, therefore, has a minimum only for $d=1$. Here we present a novel method to stabilize solitons in $2 \mathrm{D}$ by causing the interaction to oscillate rapidly using, e.g., Feshbach resonance [6, 7].

The system considered here is a BEC confined in a quasi-2D axisymmetric trap [8], where the axial confinement energy $\hbar \omega_{z}$ is much larger than the radial confinement and interaction energies. We assume that the condensate wave function $\psi$ is always in the ground state of the harmonic potential with respect to the $z$ direction, and that the dynamics are effectively $2 \mathrm{D}$ in the $x-y$ plane; we will justify these assumptions later. We let the radial confinement frequency $\omega_{\perp}(t)$ and the s-wave scattering length $a(t)$ vary in time. The system is then described by the Gross-Pitaevskii (GP) equation:

$$
i \frac{\partial \psi}{\partial t}=-\frac{1}{2} \nabla^{2} \psi+\frac{\omega_{\perp}^{2}(t)}{2} r^{2} \psi+g(t)|\psi|^{2} \psi
$$

where $r^{2} \equiv x^{2}+y^{2}$ and $g(t) \equiv\left(8 \pi m \omega_{z} / \hbar\right)^{1 / 2} N a(t)$ de- scribes the strength of interactions. In Eq. (1), length, time, frequency, and $\psi$ are measured in units of $d_{0} \equiv$ $\left[\hbar /\left(m \omega_{\perp 0}\right)\right]^{1 / 2}, \omega_{\perp 0}^{-1}, \omega_{\perp 0}$, and $N^{1 / 2} / d_{0}$, where $\omega_{\perp 0} \equiv$ $\omega_{\perp}(0)$.

We make the strength of interaction oscillate rapidly at frequency $\Omega$. The oscillation of the scattering length with $\Omega \simeq \omega_{\perp}$ is studied in Ref. [9] in a different context. Experimentally, the speed of the change in the strength of interaction using Feshbach resonance is limited by that of the applied magnetic field $\sim 1 \mathrm{G} / \mu \mathrm{s}$ 10, which is sufficient for our purpose. In order to avoid nonadiabatic disturbances that destabilize a soliton state, we gradually switch on interaction and simultaneously turn off the radial confinement potential as follows

$$
\begin{aligned}
g(t) & =f(t)\left(g_{0}+g_{1} \sin \Omega t\right), \\
\omega_{\perp}^{2}(t) & =1-f(t),
\end{aligned}
$$

where $f(t)$ is a ramp function

$$
f(t)= \begin{cases}t / T & (0 \leq t \leq T) \\ 1 & (t>T)\end{cases}
$$

The interaction and the potential can be changed independently using an optical trap and magnetic-fieldinduced Feshbach resonance.

We numerically solve the GP equation (11) with timedependent parameters (2)-(41) using the Crank-Nicholson scheme 11]. The initial state is assumed to be the noninteracting ground state in the presence of the radial confinement potential with $\omega_{\perp 0}$. We gradually increase the strength of interaction and switch off the trap according to the linear ramp (雨) with $T=20$. Figure 1 demonstrates the dynamic stabilization of a soliton in $2 \mathrm{D}$, where the parameters used are $g_{0}=-2 \pi, g_{1}=8 \pi$, and $\Omega=40$ [Fig. I (a)] and $\Omega=30$ [Fig. I (b)]. Since the value of $g_{0}=-2 \pi$ exceeds the critical strength of attractive interaction for the collapse $g_{\mathrm{cr}} \simeq-5.8$ [12], the condensate would collapse if the oscillating term $g_{1} \sin \Omega t$ were absent. Even after the radial confinement potential vanishes $(t>20)$, the soliton shape is maintained as shown in the insets of Fig. 1. The plots of the peak density $|\psi(r=0)|^{2}$ and the monopole moment $\langle r\rangle=\int r|\psi|^{2} d \mathbf{r}$ rapidly oscillate at frequency $\Omega$. We note here that the dynamics of BEC can be separated into two parts: a 

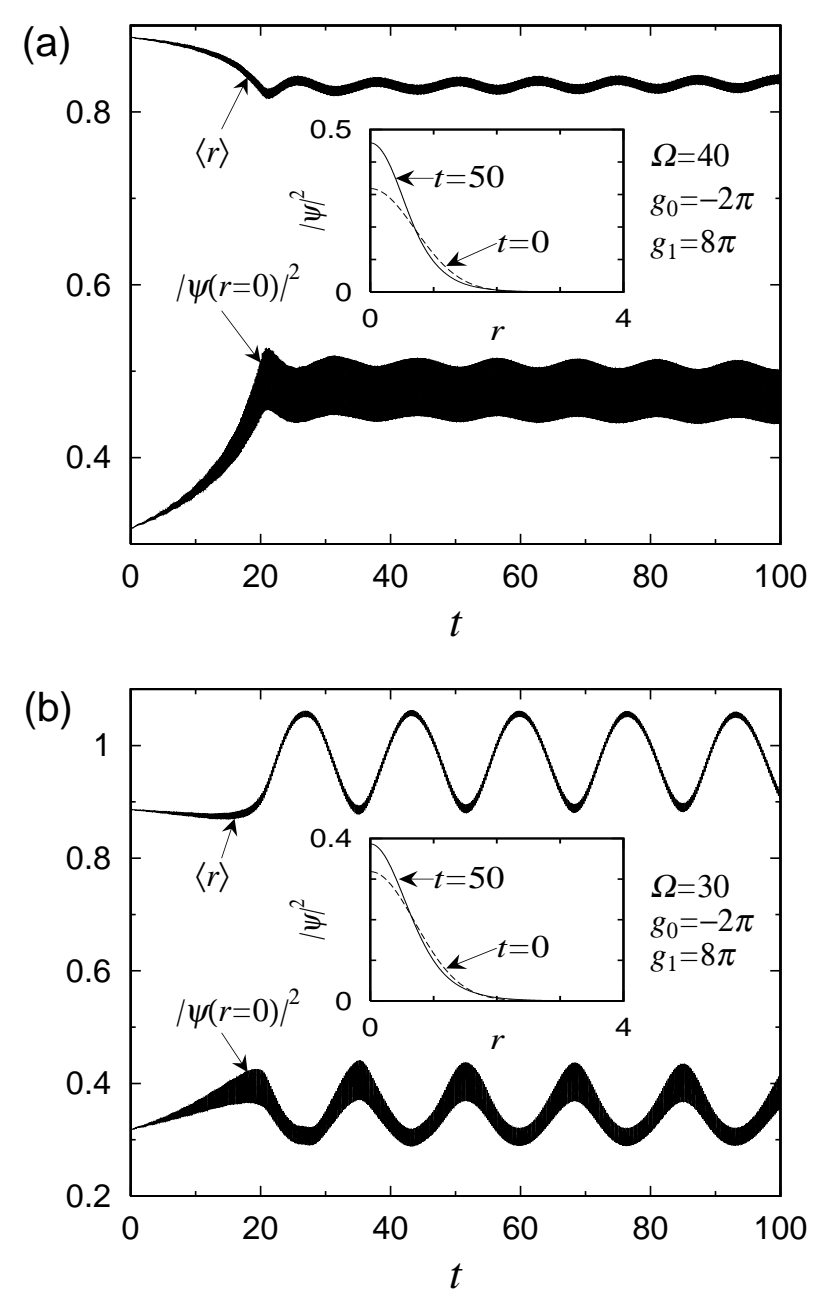

FIG. 1: Time evolution of the peak density $|\psi(r=0)|^{2}$ and the monopole moment $\langle r\rangle=\int r|\psi|^{2} d \mathbf{r}$. The interaction $g(t)=-2 \pi+8 \pi \sin \Omega t$ is switched on and the radial confinement potential is switched off from $t=0$ to 20 according to Eq. (井), where $\Omega=40$ in (a) and $\Omega=30$ in (b). The plots oscillate rapidly at $\Omega$ (which is beyond the resolution limit of the graph), and the vertical width of the plots represents the amplitude of the rapid oscillations. The insets show the density profiles $|\psi(r)|^{2}$ at $t=50$ (solid curves) and $t=0$ (dashed curves).

rapidly oscillating part with small amplitude and a slow, smoothly varying part. After the rapid oscillations are averaged out, the width and the peak density are fairly constant as seen in Fig. 11 (a). However, when the nonadiabaticity of ramp function $f(t)$ is not negligible, the lowest breathing mode (but no other radial modes) is excited as illustrated in Fig. 1 (b), where the breathing mode is caused by an effective potential due to the oscillating interaction [see Eqs. (9)-(11)]. The amplitude of the excitation decreases with increasing ramp time $T$. Such excitations can be suppressed by choosing the appropriate ramp functions for $g(t)$ and $\omega_{\perp}(t)$. We have confirmed that no symmetry-breaking mode grows in the azimuthal direction. The system is therefore free from such modulational instabilities.

We checked the validity of the quasi-2D approximation by numerically integrating the GP equation in 3D axisymmetric systems with large asymmetry parameters $\omega_{z} / \omega_{\perp} \sim 50$, and found that soliton stabilization occurs without axial modes being excited. Our prediction of dynamically stabilizing bright solitons in 2D is, therefore, experimentally feasible with an oblate trap as realized by the MIT group [8].

To understand the behavior in Fig. 1, we employ the variational method with a Gaussian wave function [9, 13, 14

$$
\psi(r, t)=\frac{1}{\sqrt{\pi} R(t)} \exp \left[-\frac{r^{2}}{2 R^{2}(t)}+i \frac{\dot{R}(t)}{2 R(t)} r^{2}\right],
$$

where $R(t)$ is the variational parameter characterizing the size of the condensate, and the imaginary term in the exponent describes mass current. Substituting Eq. (5) into the action that derives the GP equation (1) and minimizing the action with respect to $R(t)$, we obtain the equation of motion for $R(t)$ as

$$
\ddot{R}(t)=\frac{1}{R^{3}(t)}+\frac{g_{0}+g_{1} \sin \Omega t}{2 \pi R^{3}(t)},
$$

where we set $f(t)=1$. We separate $R(t)$ into the slowly varying part $R_{0}(t)$ and the rapidly oscillating part $\rho(t)$ as $R(t)=R_{0}(t)+\rho(t)$ [1]. When $\Omega \gg 1, \rho(t)$ becomes of the order of $\Omega^{-2}$, and we keep the terms of the order of up to $\Omega^{-2}$ in the following analysis. Substituting this $R(t)$ into Eq. (6), we obtain the equations of motion for the rapidly and slowly varying parts as

$$
\begin{aligned}
\ddot{\rho}(t) & =\frac{g_{1}}{2 \pi R_{0}^{3}(t)} \sin \Omega t, \\
\ddot{R_{0}}(t) & =\frac{g_{0}+2 \pi}{2 \pi R_{0}^{3}(t)}-\frac{3 g_{1}}{2 \pi R_{0}^{4}(t)} \overline{\rho(t) \sin \Omega t},
\end{aligned}
$$

where the overline indicates the time average of the rapid oscillation. Equation (7) yields $\rho(t)=$ $-g_{1} /\left[2 \pi \Omega^{2} R_{0}^{3}(t)\right] \sin \Omega t$. Substituting this into Eq. (8), we obtain the equation of motion for the slowly varying part as

$$
\ddot{R}_{0}=-\frac{\partial}{\partial R_{0}}\left(\frac{g_{0}+2 \pi}{4 \pi R_{0}^{2}}+\frac{g_{1}^{2}}{16 \pi \Omega^{2} R_{0}^{6}}\right) \equiv-\frac{\partial U\left(R_{0}\right)}{\partial R_{0}} .
$$

The minimum of the effective potential $U$ is attained at

$$
R_{\min }^{4}=-\frac{3 g_{1}^{2}}{4 \pi \Omega^{2}\left(g_{0}+2 \pi\right)}
$$

and the frequency of small oscillations (breathing mode) around the minimum is given by

$$
\omega_{\mathrm{br}}^{2}=\frac{8 \Omega^{2}}{3 g_{1}^{2}}\left(g_{0}+2 \pi\right)^{2} .
$$


(a)

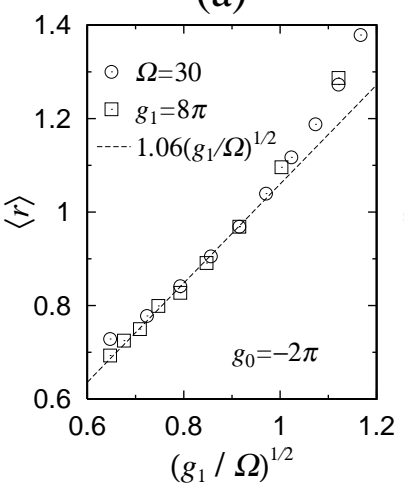

(b)

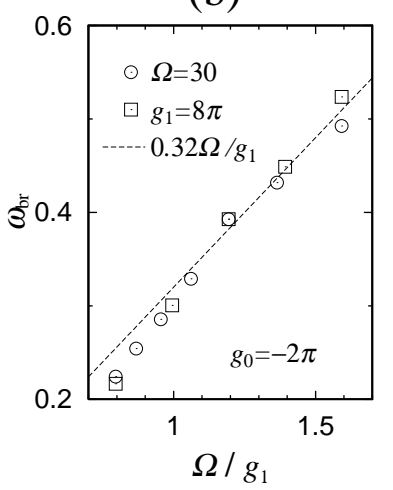

FIG. 2: (a) $\left(g_{1} / \Omega\right)^{1 / 2}$ dependence of the monopole moment $\langle r\rangle$ and (b) $\Omega / g_{1}$ dependence of the breathing-mode frequency $\omega_{\mathrm{br}}$, where $g_{0}=-2 \pi$. The circles are obtained by varying $g_{1}$ for $\Omega=30$, and the squares by varying $\Omega$ for $g_{1}=8 \pi$. The dashed lines show $1.06\left(g_{1} / \Omega\right)^{1 / 2}$ in (a) and $0.32 \Omega / g_{1}$ in (b), which are determined to best fit the plots.

The Gaussian approximation thus indicates that a soliton is stable for $g_{0}<-2 \pi$.

The physical mechanism of soliton stabilization can be understood from the above discussion. In the inverted pendulum, the interplay between the micromotion of the bob and the force gradient (i.e., stronger oscillating force for larger deviation from the equilibrium position) produces a pseudopotential. Since the pseudopotential is proportional to the square of the amplitude of the oscillating force [1], a potential barrier is formed around the equilibrium position, thereby preventing the pendulum from swinging down. Such a mechanism also stabilizes a BEC in a double-well potential with oscillating interaction [15] and an optical beam propagating in nonlinear medium with an alternating nonlinearity [16]. In the present case, the oscillating "force" for $R$ is given by $g_{1} \sin \Omega t /\left(2 \pi R^{3}\right)$, which becomes larger for smaller $R$. This force gradient and the micromotion of $R$ produce a pseudopotential proportional to the square of the "force" $\propto R^{-6}$, which prevents the system from collapsing by counteracting the $-R^{-2}$ term that describes the attractive interaction.

In order to stabilize the soliton, $\left|g_{0}\right|$ must exceed the critical value of collapse, $\left|g_{\text {cr }}\right| \simeq 5.8$, which is smaller than that of the Gaussian approximation, $\left|g_{\mathrm{cr}}\right|=2 \pi$, since the Gaussian wave function underestimates the peak density [14]. In fact, the GP equation predicts that a soliton state for $g_{0}=-2 \pi$ is stable (Fig. 1). Although the Gaussian approximation does not accurately describe the exact soliton stability condition, Eqs. (10) and (11) capture the $g_{1}$ and $\Omega$ dependences of $R_{\min }$ and $\omega_{\mathrm{br}}$. Figure 2 (a) illustrates the monopole moment $\langle r\rangle$ versus $\left(g_{1} / \Omega\right)^{1 / 2}$, where the circles are obtained by varying $g_{1}$ and the squares by varying $\Omega$. We note that the plots are fairly well proportional to $\left(g_{1} / \Omega\right)^{1 / 2}$, in agreement

(a)

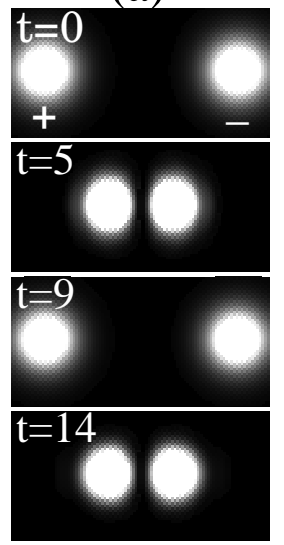

(b)

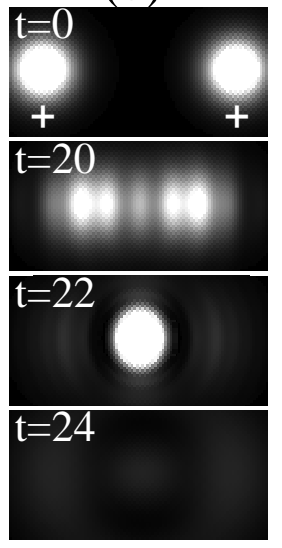

(c)

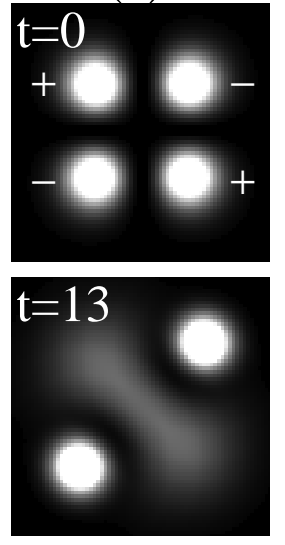

FIG. 3: Time evolution of the solitons with $g(t)=-2 \pi+$ $8 \pi \sin 40 t$, where each soliton is prepared as in Fig. 1 (a). In (a) and (c), a weak radial harmonic confinement $\omega_{\perp}^{2}=0.1 \omega_{\perp 0}^{2}$ is added. Two solitons are initially placed at $x= \pm 3$ with their phases differing by $\pi$ in (a) and with the same phase in (b). In (c), four solitons are initially placed on a slightly deformed square lattice, such that the adjacent solitons have phases differing by $\pi$. The images are $8 \times 4$ in (a) and (b), and $8 \times 8$ in (c) in units of $\left(\hbar / m \omega_{\perp 0}\right)^{1 / 2}$.

with Eq. (10). Figure 2 (b) shows the frequencies $\omega_{\text {br }}$ of the slow oscillations [as shown in Fig. [1 (b)] versus $\Omega / g_{1}$. The plots are linear in $\Omega / g_{1}$, which is consistent with Eq. (11).

The appropriate parameter range for stabilizing solitons with size $R \simeq 1$ is found to be $0.4 \lesssim g_{1} / \Omega \lesssim 1.2$ and $g_{0} \simeq-2 \pi$ for $\Omega$ much larger than the breathing-mode frequency $\omega_{\mathrm{br}}$. For $\Omega \lesssim 10$, the rapid oscillations are disturbed by nonadiabatic slow dynamics, and the soliton becomes unstable. In analogy with the inverted pendulum problem, this corresponds to a situation in which, the pendulum bob falls a long way during a single pivot cycle at a low pivot oscillation frequency. Thus, the effective force fails to stabilize the pendulum bob. When $g_{1} / \Omega$ is small or $\left|g_{0}\right|$ is large, the effective force is not sufficient to prevent the atoms from accumulating at the center. As a result, the peak density first grows, the condensate then expands due to the $R^{-6}$ potential, and subsequently most of the expanded atoms accumulate at the center due to the $-R^{-2}$ potential. Thus, the condensate repeatedly contracts and expands. In each expansion, atoms that are elastically scattered with high energy cannot return to the soliton region due to the absence of the external confinement potential, and the condensate gradually decays.

We next consider the case in which two or more solitons coexist. The effective interaction between solitons depends on their relative phase [17]. Figure 3 shows the dynamics of solitons with $g(t)=-2 \pi+8 \pi \sin 40 t$. In Figs. 3 (a) and (c), a weak radial confinement $\omega_{\perp}^{2}=0.1 \omega_{\perp 0}^{2}$ is 
added to keep the solitons from flying away. The initial states are taken to be $\psi_{\text {sol }}(x+3, y) \mp \psi_{\text {sol }}(x-3, y)$ in Figs. 3 (a) and (b), where $\psi_{\text {sol }}$ is the wave function of a single soliton prepared as in Fig. 1 (a). When two solitons have phases differing by $\pi$, they repel each other and oscillate at frequency $\simeq 2 \omega_{\perp}$ [Fig. 3 (a)]. This situation is similar to the quasi-1D experiment performed by the Rice group [5], where adjacent solitons repel each other due to the $\pi$-phase difference. When two solitons have the same phase, they approach each other without the confinement potential, and merge into one condensate, which then expands [Fig. 3 (b)].

The repulsive interaction between solitons in 2D might suggest that a stable "soliton lattice" can be formed without an external periodic potential. However, this is not the case. Figure 3 (c) shows time evolution of a "soliton lattice," where initially the adjacent solitons have phases differing by $\pi$ and their configuration is slightly perturbed to a rhombus to examine the stability against lattice distortion. There is a dynamical instability such that diagonal solitons merge, which is followed by complicated dynamics. In the presence of a periodic potential, such alternative-phase structures are shown to be both stable in 1D 18 and unstable, but can survive for a long time with appropriate parameters, in 2D and 3D [19. In order to study the stability of our system in a periodic potential, we inserted the "plug" (an external potential such as $e^{-4 r^{2}}$ ) between diagonal solitons, and found that the lattice structure survives for a long time.

In conclusion, we have demonstrated that matter-wave bright solitons can be stabilized in 2D free space by oscillating the strength of the interaction around an attractive value $g_{0}<g_{\text {cr }}$ with an amplitude $g_{1}>\left|g_{0}\right|$. The rapid oscillation of interaction produces an effective barrier that prevents the condensate from collapsing and stabilizes solitons. The interaction between solitons can be either repulsive or attractive, depending on their relative phase, and for repulsive interaction a soliton lattice can be stabilized using an optical plug. This novel technique of increasing dimensions of matter-wave bright solitons might be applied for quantum information processing using BEC solitons on a microchip substrate [20]. It merits further study to examine whether BEC "droplets" in $3 \mathrm{D}$ can be created with the appropriate parameters and ramp schemes.

This work was supported by a Grant-in-Aid for Scientific Research (Grant No. 11216204) and Special Coordi- nation Funds for Promoting Science and Technology by the Ministry of Education, Science, Sports, and Culture of Japan, by the Toray Science Foundation, and by the Yamada Science Foundation.

[1] L. D. Landau and E. M. Lifshitz, Mechanics, (Pergamon, Oxford, 1960).

[2] R. P. Feynman, R. B. Leighton, and M. L. Sands, The Feynman Lectures on Physics, (Addison-Wesley, Massachusetts, 1964), Vol. II, Chap. 29.

[3] H. G. Dehmelt, Adv. At. Mol. Phys. 3, 53 (1967).

[4] L. Khaykovich, F. Schreck, G. Ferrari, T. Bourdel, J. Cubizolles, L. D. Carr, Y. Castin, and C. Salomon, Science 296, 1290 (2002).

[5] K. E. Strecker, G. B. Partridge, A. G. Truscott, and R. G. Hulet, Nature 417, 150 (2002).

[6] S. Inouye, M. R. Andrews, J. Stenger, H. -J. Miesner, D. M. Stamper-Kurn, and W. Ketterle, Nature 392, 151 (1998).

[7] S. L. Cornish, N. R. Claussen, J. L. Roberts, E. A. Cornell, and C. E. Wieman, Phys. Rev. Lett. 85, 1795 (2000).

[8] A. Görlitz, J. M. Vogels, A. E. Leanhardt, C. Raman, T. L. Gustavson, J. R. Abo-Shaeer, A. P. Chikkatur, S. Gupta, S. Inouye, T. Rosenband, and W. Ketterle, Phys. Rev. Lett. 87, 130402 (2001).

[9] F. Kh. Abdullaev, J. C. Bronski, and R. M. Galimzyanov, cond-mat/0205464.

[10] N. R. Claussen, E. A. Donley, S. T. Thompson, and C. E. Wieman, Phys. Rev. Lett. 89, 010401 (2002).

[11] P. A. Ruprecht, M. J. Holland, K. Burnett, and M. Edwards, Phys. Rev. A 51, 4704 (1995).

[12] S. K. Adhikari, Phys. Lett. A 265, 91 (2000).

[13] V. M. Pérez-García, H. Michinel, J. I. Cirac, M. Lewenstein, and P. Zoller, Phys. Rev. Lett. 77, 5320 (1996); Phys. Rev. A 56, 1424 (1997).

[14] H. Saito and M. Ueda, Phys. Rev. A 63, 043601 (2001).

[15] F. Kh. Abdullaev and R. A. Kraenkel, Phys. Lett. A 272, 395 (2000).

[16] I. Towers and B. A. Malomed, J. Opt. Soc. Am. B 19, 537 (2002).

[17] J. P. Gordon, Opt. Lett. 8, 596 (1983).

[18] J. C. Bronski, L. D. Carr, R. Carretero-González, B. Deconinck, J. N. Kutz, and K. Promislow, Phys. Rev. E 64, 056615 (2001).

[19] B. Deconinck, B. A. Frigyik, and J. N. Kutz, J. Nonlinear Sci. 12, 169 (2002).

[20] W. Hänsel, P. Hommelhoff, T. W. Hänsch, and J. Reichel, Nature 413, 498 (2001). 